\title{
OVERVIEW ON CHITOSAN AS A VALUABLE INGREDIENT AND BIOSTIMULANT IN PHARMACEUTICAL INDUSTRIES AND AGRICULTURAL PRODUCTS
}

\author{
N. Qavami ${ }^{1}$, H. Naghdi Badi ${ }^{1}$, M.R. Labbafi ${ }^{1}$, M. Mehregan ${ }^{2}$, M. Tavakoli ${ }^{1}$, \\ A. Mehrafarin ${ }^{1} *$ \\ ${ }^{1}$ Medicinal Plants Research Centre, Institute of Medicinal Plants, ACECR, Karaj, Iran \\ ${ }^{2}$ Department of Horticulture, Science and Research Branch, Islamic Azad University, Tehran, Iran
}

\begin{abstract}
Chitosan is a polysaccharide extracted from the shells of crustaceans, such as shrimp, crab, and cell walls of fungi. Chitosan is formed from chitin, a co-polymer of $N$-acetyl- $D$-glucosamine and $D$ glucosamine, when over $80 \%$ of the acetyl groups of the $N$-acetyl- $D$-glucosamine residues are removed. Chitosan and its derivatives have been shown to possess diverse biological activities, including antioxidant, anti-hypertensive, anti-coagulant, anti-diabetic, anti-obesity, anti-allergic, antiinflammatory, anti-microbial, and anti-cancer. Chitosan has been studied in recent years as a natural agrochemical biostimulant for applications in areas such as seed coating, preservation of fruits and vegetables, fungicides, growth biostimulants, elicitors, inducer of resistance to pathogens, etc. Chitosan as a biostimulant has biological properties and effective uses in the pharmaceutical industries, food industries, and agricultural products. This paper is a brief review on characterization, modification, and applications of chitosan.
\end{abstract}

Keywords: Chitosan, Biological activity, Pharmacological application, Natural biostimulant

\section{INTRODUCTION}

Chitosan, a natural polysaccharide and biopolymer, is produced by alkaline deacetylation of chitin, which is derived from crustaceans such as cray fish, lobster, prawns, crab and shrimp (1-3). Chitosan also occurs naturally in some microorganisms such as fungi and yeast $(4,5)$ and is considered the largest biomaterial after cellulose in terms of utilization and distribution $(1,5)$. Its chemical name is 2 -amino-2-deoxy- $b$ - $D$-glucopyranose and its formula is $\left(\mathrm{C}_{6} \mathrm{H}_{11} \mathrm{O}_{4} \mathrm{~N}\right)(2)$.

The development of commercial applications for chitosan has progressed (6). Chitin and chitosan are produced commercially in India, Poland, Japan, Thailand, USA, Norway and Australia $(7,8)$. Chitosan has three types of reactive functional groups, an amino/acetamido group as well as both primary and secondary hydroxyl groups at the C-2, C-3, and C-6 positions, respectively. The

\footnotetext{
*Correspondence to: A. Mehrafarin, Medicinal Plants Research Centre, Institute of Medicinal Plants, ACECR, P.O.Box:,33651/66591Karaj, Iran Tel: +98-26-34764010-9, Fax: +98-26-34764021 Email: A.mehrafarin@gmail.com
}

amino contents are the main factors contributing to the differences in their structures and physico-chemical properties (9).

The chitosan has attracted considerable interest in various fields due to its unique biological activities, such as biocompatibility $(10,11)$, biodegradability (12), nontoxicity (13), antimicrobial activity $(14,15)$, antitumor activity (16) and immune-enhancing effect (17, 18). These properties make chitosan a promising candidate for medicine (19), food $(7,20)$, cosmetic (21), water treatment (22), biomedical engineering industries $(23,24)$ and many agricultural uses (25-27). The purpose of this paper is to highlight the application of this polysaccharide that is gaining much interest due to the properties it presents and the many applications in various fields.

\section{Sources of chitosan}

Chitosan is commercially produced from deacetylated chitin, which is the most abundant polysaccharide in nature following cellulose (28). Chitosan can be extracted from insects, yeast, mushroom, cell wall of fungi, and marine shellfish such as crab, lobster, krill, 
cuttlefish, shrimp, and squid pens (8). Fungal chitosan gives medium-low molecular weight chitosans, whereas the molecular weight of chitosans obtained from crustacean sources is high. Chitosan with a medium-low molecular weight has biomedical application $(6,29)$.

\section{Chemical structure}

Chitosan is a linear polysaccharide consists of D-glucosamine linked to $N$-acetyl- $D$ glucosamine by $\beta$-1,4-glycosidic bond (Figure 1) (2). The distribution of these subunits depends on the method of preparation of chitosan. In chitosan, degree of deacetylation ranges from 40 to $98 \%$ and the molecular weight ranges5-10 ${ }^{4} \mathrm{Da}$ (Dalton (Da) is the
QAVAMI N., et al. standard unit that is used for indicating molecular weight) and $2-10^{6} \mathrm{Da}(8,30)$. Chitin and chitosan are insoluble in water as well as most organic solvents, which is the major limiting factor for their utilization in living systems (9) but it is soluble in acid solutions. Solubility of chitosan is related to the degree of deacetylation, the ionic concentration, $\mathrm{pH}$, nature of the acid used for protonation and the distribution of acetyl groups along the chain (8). Most of the properties of chitosan can be related to its cationic nature $(9-12)$, which is unique among abundant polysaccharides and natural polymers (31).

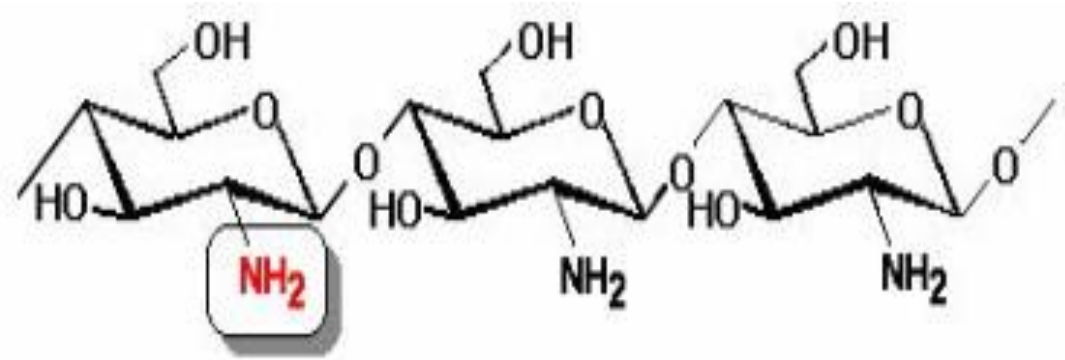

Figure 1. Chemical structure of chitosan (2)

\section{Production of chitosan}

Typically, chitosan is obtained by deacetylation of the $\mathrm{N}$-acetyl glucosamine units of chitin, under alkali conditions at high temperature (30). The degree of deacetylation (\%DD) can be determined by NMR spectroscopy (Nuclear magnetic resonance spectroscopy is a research technique that determines the physical and chemical properties of atoms or the molecules.), and the $\%$ DD in commercial chitosans ranges from 60 to $100 \%$ (2). The main parameters influencing the characteristics of chitosan are its degree of deacetylation (DD) and molecular weight $(\mathrm{Mw})$, which affect the solubility and physical properties (6). Different conditions such as type and concentration of reagents, time and temperature employed throughout the processing can affect the physical characteristics and performance of the final chitosan product (32). The production of chitosan involves a two-step process. The first step is extraction of chitin and removal of calcium carbonate $\left(\mathrm{CaCO}_{3}\right)$ from crustacean's shells using dilute hydrochloric acid and deproteination with dilute aqueous $\mathrm{NaOH}$. In the second step, $40-50 \%$ aqueous $\mathrm{NaOH}$ is used for deacetylation of chitin for several hours without oxygen. When the degree of deacetylation exceeds $50 \%$, then chitosan is produced (Figure 2) $(5,33,34)$.
Chitosan is the $\mathrm{N}$-deacetylated form of chitin and linear polysaccharides with a variable degree of $N$-acetylation, which is composed of less than $20 \% \quad b$-(1,4)-2-acetamido- $D$ glucopyranose and more than $80 \% \quad b$ - $(1,4)-2-$ amino- $D$-glucopyranose.Chitosan is waterinsoluble and highly viscous in dilute acidic solutions. This solubility drawback may restrict the applications of chitosan in biological fields. In contrast, as the hydrolyzed products of chitosan, and oligosaccharides have better solubility and lower viscosity under physiological conditions because of shorter chain lengths and free amino groups in $D$ glucosamine units (28). Chitin and chitosan are biodegradable biopolymers. Enzymes (chitinase and chitosanase) break them down into oligopolymers that are then dealt with by the metabolism.

\section{APPLICATIONS OF CHITOSAN}

Chitosan and its derivatives have varied applications in agriculture, food processing, biotechnology, chemistry, cosmetics, dentistry, medicine, textiles, veterinary sciences, and environmental sciences. The polyelectrolyte nature and the presence of reactive functional groups are responsible for the gel-forming ability, high adsorption capacity, biodegradability, and antimicrobial properties of chitosan which in turn are essential for its commercial applications (8). 


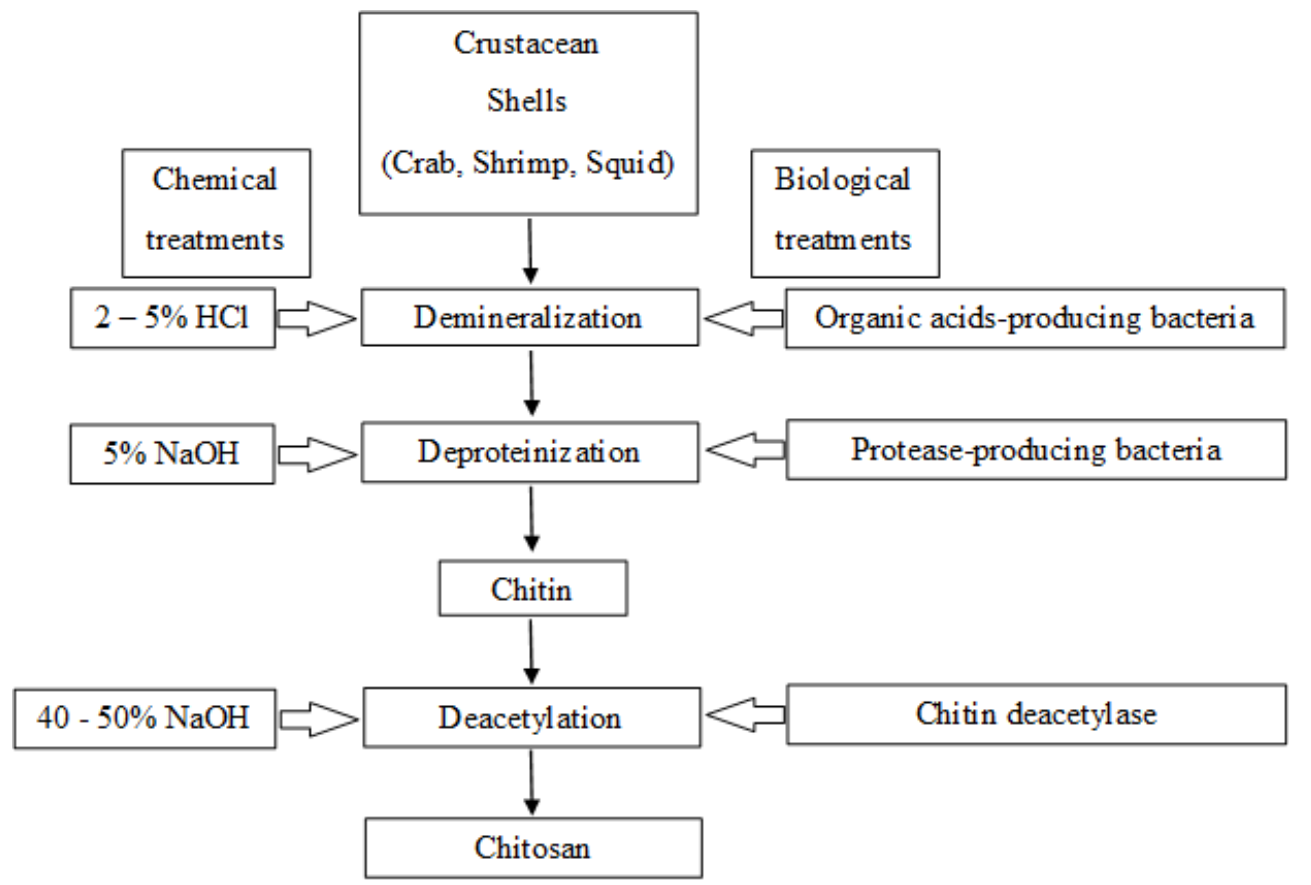

Figure 2. Chitin and chitosan production by chemical and biological treatments (34)

\section{1- BIOMEDICAL APPLICATIONS}

Chitosan, due to its remarkable physicochemical and biological properties, is one of the most promising polymers for biomedical applications (35). It is used in artificial skin, hemostatic agents, and drug delivery systems (8).

\section{1-1- Drug delivery carriers}

The chemical modification of chitosan is a powerful tool to control the interaction of the polymer with drugs, enhance the load capability, and organizer the release profile of the drug carriers. Drug delivery carriers must not accumulate in the body nor must it be toxic $(6,36)$. Chitosan has ability to control the release of active agents and avoid the use of hazardous organic solvents. Chitosan in the form of colloidal structures can entrap macromolecules by various mechanisms. These associated macromolecules have been shown to transport through mucosa and epithelia more efficiently $(6,37)$. Chitosan is considered the most important polysaccharide for various drug delivery purposes because of its cationic character and primary amino groups(5). For effective drug delivery, it is being used in the form of microspheres, micro particles, nanoparticles, granules, gels, or films. Chitosan's microspheres are useful for the controlled release of antibodies, antihypertensive agents, anticancer agents, protein and peptide drugs, vaccines, and nutraceutical compounds (38)

\section{1-2- Wound healing agent}

Due to bacteriostatic and fungistatic properties of chitosan, it is used as a wound healing agent in skin ointments. Implanted chitosan in animal tissues encourages wound healing. Chitosan has been studied widely as a wound dressing material. Burkatovskaya et al. (39) has found that the chitosan acetate bandage provided important benefits by reducing the number of inflammatory cells in the mice wounds (5). Biocompatible wound dressings derived from chitosan are available in the forms of hydrogels, xerogels, powders, composites and film (2). Studies have been shown that chitosan has a hemostatic effect (40). Hemostasis through blood coagulation is an important step for wound healing. Okamoto et al. (41), reported that chitin is an effective agent for hemostasis and expressed that the effect of chitin and chitosan is due to both physical and chemical properties of these biopolymers especially their amino groups.

\section{1-3- Scaffold for the regeneration of tissue}

Chitosan forms films that are permeable to air. It facilitates cellular regeneration while protecting tissue from microbe attack. In addition, chitosan has been found to have a biostimulant effect on the regeneration of tissue. This property has allowed it to be used in making an artificial skin for skin grafts on high degree burns and in surgical applications such as chitin suture thread. Due to its remarkable properties, chitosan has been applied to the synthesis of scaffolds or hydrogels dedicated to tissue engineering (30). A good scaffold should have at least four important factors: (1) ability to form temporary matrix, (2) ability to form porous structure for tissue to grow, (3) biodegradability and finally 
(4) nontoxic byproducts from the digestion (6, $42,43)$.

\section{1-4- Artificial skin}

It appears that chitosan polysaccharides having structural characteristics similar to glycosaminoglycans can be considered for developing such substratum for skin replacement (7). Dressing materials based on chitin, chitosan and derivatives are well-known on the market, and are produced mainly in Japan and the USA (6).

\section{2-PHARMACOLOGICAL APPLICATIONS}

\section{2-1- Antitumor activity}

The antitumor activity of chitin/chitosan is manifested by the stimulation of the immune system $(6,44)$. Studies showed that novel chitosan-thymine conjugate has been successfully synthesized by the acylation reaction between chitosan and thymine-1-ylacetic acid. The assays for cell proliferation and viability showed that the chitosan-thymine conjugate was non-cytotoxic but significantly reduced the rate of proliferation in cancerous $\mathrm{HepG}_{2}$ cells (45). Thus, the chitosan-thymine conjugate might be a very promising candidate for practical applications in the field of biomedicine(46). Salah et al. (47) evaluated the anticancer abilities of chitin, chitosan and low molecular weight chitin using a human tumor cell line THP-1. The results indicated that low molecular chitin had a higher tumorsuppressive activity and the tumor suppression increased significantly with the decrease of the molecular weight (28).

\section{2-2- Blood cholesterol control}

Chitosan can trap lipids at their insolubilization $\mathrm{pH}$ in the digestive tract. Administered to rats, chitosan considerably reduces the level of cholesterol in the blood. The hypocholesterolemic effect of chitosan has also been found in humans (48). It reduces lipid absorption by binding neutral lipids, such as cholesterol and other sterols, by means of hydrophobic interactions. Because of this inhibitory activity on fat absorption, chitosan acts as fat scavenger in the digestive tract and eliminates fat and cholesterol via excretion $(8$, 49).

\section{2-3- Anti-coagulants}

Blood coagulation is processed by coagulation factors in order to stop the flow of blood at sites of vascular injury occur. Chitosan sulfates have been shown anti-coagulant activity. Vongchan et al. (50) studied anti-coagulants effect of three sulfated chitosan and express these preparations showed strong anticoagulant activities, with the same mechanism of action observed for standard therapeutic heparin (2, $50)$.

\section{2-4- Antimicrobial properties of chitosan}

Chitosan exhibits a variety of antimicrobial activity (15), against bacteria, molds and yeasts(8). In 1979, the first study reported that chitosan showed a broad range of activities and a high inactivation rate against both grampositive and gram-negative bacteria $(27,51)$. Chitosan antimicrobial activity depends on its molecular weight, degree of deacylation, the host, the chemical and/or nutrient composition of the substrates, and environmental conditions. The exact mechanism of antibacterial activity of chitosan is not fully understood (8). Antiviral results of the chitosan demonstrated that it could replace chemical pesticide to prevent and control infection of economic insect by viral pathogens, avoiding environmental pollution (2).

\section{2-5- Antioxidant activity}

Chitosan and its derivatives have been reported to have strong antioxidant activity. They are well known in scavenging free radicals or in preventing oxidative damage by interrupting the radical chain reaction of oxidation, since they can protect human body against deterioration by free radicals (9). They control lipid oxidation by scavenging free radicals, which can be attributed to their ability to chelate metals. Anraku et al. (52) investigated antioxidant effect of high molecular weight chitosan supplement (chitosamin) in normal human volunteers. Chitosamin decreased lipid hydro peroxides and uremic toxins in the gastrointestinal tract, thereby inhibiting the subsequent development of oxidative stress in the systemic circulation in human. Thus, the anti-oxidative effect of chitosamin is unique and differs from that of typical, conventional antioxidants such as antioxidant vitamins and $\mathrm{N}$-acetylcysteine. The antioxidant effects of chitin and chitosan are dependent on their molecular weight, viscosity, and degree of deacetylation $(8,53)$.

\section{2-6- Side effects and interactions}

While no long-term studies of the effects of chitosan on human health have been done, animal studies suggest that this compound could inhibit the absorption of minerals and fat-soluble vitamins. Although chitosanincluded alterations in intestinal flora are believed to be beneficial, the possibility that these changes may have negative long-term consequences has not been ruled out. People 
with intestinal malabsorption syndromes should not use chitosan. Information regarding safety and efficacy in pregnancy and lactation is lacking. The potential for allergy exists in individuals allergic to shellfish. Clinical trials report few adverse events, generally limited to flatulence and constipation (2).

\section{3-OTHER APPLICATIONS OF CHITOSAN}

\section{3-1- Application in agriculture}

Chitosan, due to the antifungal, antibacterial and antiviral properties, it has been used successfully in agriculture in recent years such as: in plant protection (seed coating, leaf coating fertilizer), plant growth promoter, in soil correction, improver of secondary metabolites production and activator of defense mechanisms (6). Chitosan also have potential in agriculture with regard to controlling plant diseases. They are active against soil fungi, viruses, bacteria, and other pests. Fragments from chitin and chitosan are known to have eliciting activities for a variety of defense responses in host plants, including the accumulation of phytoalexins, pathogenrelated (PR) proteins, proteinase inhibitors, lignin synthesis $(8,26)$. Chitosan increases photosynthesis, promotes and enhances plant growth, stimulates nutrient uptake, increases germination and sprouting, and boosts plant vigor (2).

Chitosan is often used in plant disease control as a powerful elicitor rather than a direct antimicrobial or toxic agent. Its direct toxicity remains dependent on properties such as the concentration applied, the molecular weight, and degree of acetylation, solvent, $\mathrm{pH}$ and viscosity (26). Because of its biopolymer properties, this compound can also form physical barriers around the penetration sites of pathogens, preventing them from spreading to healthy tissues (2).

Chitin derivatives can have actions outside living cell, as polycationic and lipid-binding molecules. This leads to applications for water cleaning, but the formation of chitosan films on the waxy surface of plant leaves prompts their use as antitranspirant. Chitosan works as both film-forming compound and physiological regulator of stomata via the ABA-dependent pathway. Some film-forming compounds, like chitosan increase leaf surface reflectance, reducing absorption of radiant energy (heat), lowering leaf temperature, reducing water evaporation within the leaf and its diffusion to the surrounding atmosphere (referred to as transpiration). Stomata are the main route of water vapor export during plant growth. When emulsions of the synthetic compounds are sprayed on leaf surface, they form thin films and limit gas exchange by increasing stomatal resistance to the diffusion of water vapor (54).

\section{3-2-Application as edible films}

The edible films and coatings are used to extend shelf life and improve quality of food products. Chitosan forms tough, long-lasting, flexible, semipermeable films that can be used as food wraps for extending their shelf life. Chitosan film could be used for extension of shelf life of bread by retarding starch retrogradation and/or by inhibiting microbial growth, extending the shelf life of eggs, prolong storage life and control decay of fruits, meat, etc. (6).

\section{3-3- Chitosan as a biostimulant in medicinal plants production}

Chitosan-based materials exhibit various interesting properties, which make them applicable in many fields, including agriculture, where they are used as biostimulants. Chitosan induces several defensive genes in plants, such as pathogenesis-related genes, like glucanase and chitinase. It also induces many enzymes in the reactive oxygen species scavenging system, such as superoxide dismutase, catalase and peroxidase. The signal transduction pathway from chitosan that elicits its responses involves phenylalanine ammonia lyase (PAL), hydrogen peroxide and nitric oxide signals, and it may also directly control gene expression by interacting with chromatin $(54,56)$.

Chitosan is frequently used as an elicitor and biostimulant to stimulate the production of active/drug compounds both in plant and in vitro systems (55). Chitosan elicitation has been also adopted as an effective strategy to enhance secondary metabolite production, such as xanthones and other polyphenols, in Hypericum perforatumin vitro roots $(56,57)$. The effects of chitosan on artemisinin accumulation in Artemisia annua hairy root cultures have been investigated. Putalon et al. (58) showed that artemisinin production in hairy roots was increased 6-fold by adding 150 mg chitosan. Study of effects chitosan elicitation on secondary metabolism of Hypericum perforatum in vitro roots showed that in response to chitosan elicitation, the biomass growth was arrested and valine, isoleucine, glutamine, $c$-aminobutyric acid, fructose, sucrose, poly unsaturated fatty acids, 
epi-catechin, xanthones, dimethyl allylpyrophosphate levels increased (57). Chitosan has been used both as a biostimulant to stimulate plant growth, and abiotic stress tolerance, and as to induce pathogen resistance; however, these responses are complex and they depend on different chitosan-based structures and concentrations as well as the plant species and developmental stage.

\section{3-4- Water filtration}

Water gets polluted due to metal ions, inorganic anions, phenolic compounds, dyes, and radioactive isotopes. Many of these water pollutants are toxic and can enter the human food chain. Chitosan is being successfully used in water treatment to remove lead, copper, and cadmium from drinking water, due to complex formation between the amino group and heavy metal ions $(8,22)$. Its use as a bio-sorbent is justified by two important advantages: firstly, its low cost compared to commercial activated carbon (chitosan is derived by deacetylation of the naturally occurring bio-polymer chitin which is the second most abundant polysaccharide in the world after cellulose); secondly, its outstanding chelation behavior (one of the major applications of this aminopolymer is based on its ability to tightly bind pollutants, in particular heavy metal ions) (31).

\section{3-5- Application in paper industry}

Biodegradable chitin and chitosan can strengthen recycled paper and increase the environmental friendliness of packaging and other product. Chitosan is already involved in the manufacture of paper because chitosan molecules greatly resemble those of cellulose the fain constituent of plant walls. The paper produced with chitosan has a smoother surface and is more resistance to moisture (7).

\section{CONCLUSION}

Chitosan is a very important biomaterial that is readily available and can be used in various fields such as agriculture, biotechnology, food processing, pharmacology and therapeutic activities.Due to the antifungal, antibacterial and antiviral properties of chitosan, it has been used successfully in agriculture in recent years, in plant protection, like growth promoter, in soil correction, enhancer of secondary metabolites production, and activator of defense mechanisms. Literature reviews have shown that the chitosan is frequently used as an elicitor and biostimulant in plants to stimulate the production of active/drug compounds.

\section{REFERENCES}

1. Mincea, M., Negrulescu, A., and Ostafe, V., Preparation, modification, and applications of chitin nanowhiskers: a review, Reviews on Advanced Materials Science, 30: 225242, 2012.

2. Gavhane Yogeshkumar, N., Gurav Atul, S., and Yadav Adhikrao, V., Chitosan and its applications: A review of literature,International Journal of Research in Pharmaceutical and Biomedical Science, 4(1): 312-331, 2013.

3. Jun, C., Jin, L., Changgao, W., Jianguo, L., Ying, H., Jianhong, Y., Yumin, D., and Hua, Z., Parametric optimization of extracellular chitin deacetylase production by scopulariopsis brevicaulis, Journal of Biocatalysis \& Biotransformation, 2(1): 15, 2013.

4. Illum, L., Jabbal-Gill, I., and Hinchcliffe, M., Chitosan as a novel nasal delivery system for vaccines, Advanced Drug Delivery Review, 51: 81-96, 2001.

5. Elgadir, MA., Uddin, MS., Ferdous, S., Adam, A., Chowdhury, A., and Islam Sarker, M.Z., Impact of chitosan composites and chitosan nanoparticle composites on various drug delivery systems: A review,Journal of Food and Drugs Analysis, 1-11, 2014.

6. Miranda Castro, S.P., and Eva,G., Is chitosan a new panacea? areas of application, in the complex world of polysaccharides. Nedra Karunaratne D, Editor., INTECH Open Access. p. 3-46., 2012.

7. Kumar Dutta, P., Dutta, J., and Tripathi, V.S., Chitin and chitosan: Chemistry, properties and application,Journal of scientific and Industrial Research, 63: 2031, 2004.

8. Chawla, S.P., Kanatt, S.R., and KSA, Chitosan in polysaccharides, Bioactivity and Biotechnology. Kishan, G., and JeanMichel, M., Editors. Springer International Publishing. p. 220-243, 2015

9. Ngo, D.H.,Vo, T.S., Ngo,D.N., Kang,K.H., Je,J.Y., Pham,H.N.D., Byun,H.G., and Kim, S.K., Biological effects of chitosan and its derivatives, Food Hydrocolloids, 51: 200-216, 2015.

10.Hsu, S.H., Chang, Y., Tsai, C.L., Fu, K.Y., Wang, S.H., and Tseng, H.J., Characterization and biocompatibility of chitosan nanocomposites, Colloids and Surfaces B: Biointerfaces, 85(2): 198-206, 2011.

11.Mi, F., Tan, Y., Liang, H., and Sung, H., In vivo biocompatibility and degradability of a novel injectable-chitosan-based 
implant,Biomaterials, $\quad$ 23(1): 181-191, 2002.

12.Kim, H., Tator,C.H., and Shoichet, M.S., Chitosan implants in the rat spinal cord: biocompatibility and biodegradation,Journal of Biomedical Materials Research, 97A(4): 395-404, 2011.

13.Shi, Z.L., Neoh,K.G., Kang, E.T., and Wang, W., Antibacterial and mechanical properties of bone cement impregnated with chitosan nanoparticles,Biomaterials, 27(11): 2440-449, 2006.

14.Li, B., Wang,X., Chen, R., Huangfu, W.G., and Xie, G., Antibacterial activity of chitosan solution against Xanthomonas pathogenic bacteria isolated from Euphorbia pulcherrima,Carbohydrate Polymers, 72(2): 287-292, 2008.

15.Rabea, E.I., Badawy, M.E.I., Steurbaut, W., and Stevens, C.V., In vitro assessment of $\mathrm{N}$-(benzyl)chitosan derivatives against some plant pathogenic bacteria and fungi,European Polymer Journal, 45(1): 237-245, 2009.

16.Toshkova, R., Manolova,N., Gardeva, E., Ignatova,M., Yossifova, L., Rashkov,I., and Alexandrov, M., Antitumor activity of quaternized chitosan-based electrospun implants against graffi myeloid tumor,International Journal of Pharmaceutics, 400(1-2): 221-233, 2010.

17.Li, X., Min, M., Du, N., Gu, Y., Hode, T., Naylor, M.,Chen, D., Nordquist, R.E., and WR, C., Chitin, chitosan, and glycated chitosan regulate immune responses: The novel adjuvants for cancer vaccine, Clinical and Developmental Immunology, 2013: 1-8, 2013.

18.Zaharoff, D.A.,Rogers, C.J., Hance, K.W., Schlom, J., and Greiner, J.W., Chitosan solution enhances both humoral and cellmediated immune responses to subcutaneous vaccination, Vaccine, 25: 2085-2094, 2007.

19.Tan, H Ma, R., Lin, C., Liu, Z., and Tang, T., Quaternized Chitosan as an Antimicrobial Agent: Antimicrobial Activity, Mechanism of Action and Biomedical Applications in Orthopedics,International Journal of Molecular Sciences, 14(1): 1854-1869, 2013.

20.Qiu, M.W.C., Ren, G., Liang, X., Wang, $\mathrm{X}$., and Huang, J., Effect of chitosan and its derivatives as antifungal and preservative agents on postharvest green asparagus, Food Chemistry, 155: 105-111, 2014.
21.Ray, S., Potential aspects of chitosan as pharmaceutical excipient,Acta poloniae pharmaceutica, 68: 619-622, 2011.

22.Bhatnagar, A., and Sillanpaa, M., Applications of chitin-and chitosanderivatives for the detoxification of water and wastewater - a short review,Advances in Colloid and Interface Science, 152: 2638, 2009.

23.Silva, T.H., Alves, A., Ferreira, B.M., Oliveira, J.M., Reys, L.L., Ferreira, R.J.F., Sousa, R.A., Silva, S.S., Mano, J.F., and Reis, R.L., Materials of marine origin: a review on polymers and ceramics of biomedical interest,International Materials Reviews, 57: 276-306, 2012.

24.Upadhyaya, L.S.J., Agarwal, V., and Tewari, R.P., Biomedical applications of carboxymethyl chitosans, Carbohydrate Polymer, 91(52-466), 2013.

25.Cota-Arriola, O., Cortez-Rocha, M., Burgos-Hernández, A., EzquerraBrauer, J., and Plascencia-Jatomea, M., Controlled release matrices and micro/nanoparticles of chitosan with antimicrobial potential: development of new strategies for microbial control in agriculture,Journal of Science Food Agriculture, 93: 1525-1536, 2013.

26.El-Hadrami, A., Adam,L.R., El-Hadrami, I., and Daayf, F., Chitosan in plant protection.Marine Drugs. 8: 968-987, 2010.

27.Xing, K., Zhu, X., Peng, X., and Qin, S., Chitosan antimicrobial and eliciting properties for pest control in agriculture: a review.Agronomy for Sustainable Development. 35(2): 569-588, 2015.

28.Zou, P., Yang,X., Wanga, J., Li, Y., Yu, H., Zhang, Y., and Liu, G., Advances in characterisation and biological activities of chitosan and chitosan oligosaccharides,Food Chemistry, 190: 1174-1181, 2016.

29.New, N., and Stevens,W.F., Chitosan isolation from the chitosan-glucan complex of fungal cell wall using amylolytic enzymes,Biotechnology Letters, 24: 14611464, 2002.

30.Riva, R., Ragelle, H., Rieux, A., Duhem, N.J., Jerom,C., and Preat, V., Chitosan and chitosan derivatives in drug delivery and tissue engineering,Advances in Polymer Science, 244: 19-44, 2011.

31.Crini, G., and Badot, P.M., Application of chitosan, a natural aminopolysaccharide, for dye removal from aqueous solutions by adsorption processes using batch studies: A review of recent literature,Progress in Polymer Science, 33: 399-447, 2008. 
32.Khan, W., Prithiviraj,B., and Smith, D.L., Chitosan and chitin oligomers increase phenylalanine ammonia-lyase and tyrosine ammonia-lyase activities in soybean leaves,Journal of Plant Physiology, 160: 859-863, 2003.

33.Steenkamp, G.C., Keizer, K., Neomagus, H.W.J.P., and Krieg, H.M., Copper (II) Removal from polluted water with alumina/chitosan composite membranes.Journal of Membrane Science. 197: 147-156, 2002.

34.Jo, G.H., Park, R.D., and Jung, W.J.,Enzymatic production of chitin from crustacean shell waste, in chitin, chitosan, oligosaccharides and their derivatives: Biological activities and applications, K. SK, Editor. CRC Press. p. 37-43, 2010.

35.Balan, V., and Verestiuc, L., Strategies to improve chitosan hemocompatibility: A review,European Polymer Journal, 53: 171-188, 2014.

36.Dev, A., Mohan,J.C., Sreeja, V., Tamura, H., Patzke, G.R., Hussain, F., Weyeneth, S., Nair, S.V., and Jayakumar,R., Novel carboxymethyl chitin nanoparticles for cancer drug delivery applications,Carbohydrate Polymer, 79: 1073-1079, 2010.

37.Janes, K.A., Calvo,P., and Alonso, M.J., Polysaccharide colloidal particles as delivery systems for macromolecules,Advanced Drug Delivery Review, 47: 83-97, 2001.

38.Dash, M., Chiellini, F., Ottenbrite, R.M., and Chiellini, E., Chitosan - a versatile semi-synthetic polymer in biomedical applications, Progress in Polymer Science, 36: 981-1014, 2011.

39.Burkatovskaya, M.,Castano, A.P., Demidova-Rice, T.N., Tagos, G.P., and Hamblin, M.R. Effect of chitosan acetate bandage on wound healing in infected and noninfected wounds in mice, Wound Repair and Regeneration, 16: 425-431, 2008.

40.Klokkevold, P.R.,Fukayama,H., Sung, E.C., and Bertolami, C.N.,The effect of chitosan (poly-N-acetyl glucosamine) on lingual hemostasis in heparinized rabbits,Journal of Oral and Maxillofacial Surgery, 57: 49-52, 1999.

41.Okamoto, Y., Yano, R., Miyatake, K., Tomohiro, I., Shigemasa, Y., and Minami, S., Effects of chitin and chitosan on blood coagulation,Carbohydrate Polymer, 2003; 53: 337-342.

42.Drury, J.L., and Mooney, D., Hydrogels for tissue engineering: scaffold design variables and applications, Biomaterials, 24: 4337-4351, 2003.
43.Khor, E.L.L. Implantable applications of chitin and chitosan, Biomaterials, 24: 23392349, 2003.

44.Qin, C., Du, Y., Xiao, L., Li, Z., and Gao, $\mathrm{X}$., Enzymic preparation of water-soluble chitosan and their antitumor activity,International Journal of Biological Macromolecules, 31: 111-117, 2002.

45.Kumar, S., Kim, H., Gupta, M.K., Dutta, P.K., and Koh, J., A new chitosan-thymine conjugate: synthesis, characterization and biological activity,International Journal of Biological Macromolecules, 50(3): 493$502,2012$.

46. Kumar, S., and Koh, J., Physiochemical, optical and biological activity of chitosanchromone derivative for biomedical applications,Int. J. Mol. Sci, 13(5): 61026116, 2012.

47.Salah, R., Michaud, P., Mati, F., Harrat, Z., Lounici, H. and Abdi, N., Anticancer activity of chemically prepared shrimp low molecular weight chitin evaluation with the human monocyte leukaemia cell line, THP1,International Journal of Biological Macromolecules, 52: 333-339, 2013.

48.Maezaki, Y., Tsuji, K., Nakagawa, Y., Kawai, Y., Akimoto, M., Tsugita, T., Takekawa, W., Terada, A., Hara, H., and Mitsuoka, T., Hypocholesterolemic effect of chitosan in adult males,Bioscience, Biotechnology, and Biochemistry, 57: 14391444, 1993.

49.Luo, Y.,and Wang, Q., Recent advances of chitosan and its derivatives for novel applications in food science,Journal of Food Process Beverage, 1(13): 1-8, 2013.

50. Vongchan, P., Sajomsang, W., Subyen, D., and Kongtawelert, P., Anticoagulant activity of a sulfated chitosan, Carbohydrate Polymer, 337(13): 1239-1242, 2002.

51.Allan, C.R., and Hadwiger, L.A., The fungicidal effect of chitosan on fungi of varying cell wall composition, Experimental Mycology, 3: 285-287, 1979.

52.Anraku, M., Fujii, T., and Kondo, Y., Antioxidant properties of high molecular weight dietary chitosan in vitro and in vivo, Carbohydrate Polymer, 83: 501-505, 2011.

53. Venugopal, V.Marine polysaccharides: food applications,CRC Press: USA. p. 396., 2011.

54. Du Jardin, P., The science of plant biostimulants - A bibliographic analysis. AD Hoc Study Report (30-CE0455515/0096) to the European Commission DG ENTR. p. $\quad 37, \quad 2012$ http://ec.europa.eu/enterprise/sectors/chemi 
cals/files/fertilizers/final report bio 2012en.pdf.

55.Yin, H., Zhao, X., and Du, Y.G., Oligochitosan A plant diseases vaccine-a review,Carbohydrate Polymer, 82: 1-8, 2010.

56.Tocci, N., D’Auria, F.D., Simonetti, G., Panella, S., Palamara, A.T., Debrassi, A., Debrassi, A., Rodriques, C.A., Filho, V.C., and Pasqua, G., Bioassay-guided fractionation of extracts from Hypericum perforatum in vitro roots treated with carboxymethylchitosans and determination of antifungal activity against human fungal pathogens,Plant Physiology and Biochemistry, 70: 342-347, 2013.
57.Brasili, E., Pratico, G., Marini, F., Valletta, A., Capuani, G., Sciubba, F., Miccheli, A., and Pasqua, G., A non-targeted

metabolomics approach to evaluate the effects of biomass growth and chitosan elicitation on primary and secondary metabolism of Hypericum perforatum in vitro roots,Metabolomics, 10(6): 11861196, 2014.

58.Putalun, W., Luealon, W., De-Eknamkul, W., Tanaka, and H., Shoyama, Y., Improvement of artemisinin production by chitosan in hairy root cultures of Artemisia annua L.,Biotechnology Letters, 29: 11431146, 2007. 